\title{
溴呋啶及其类似物的合成新方法研究
}

\author{
李培源张建瑞郭胜海张新迎* 范学森* \\ (河南师范大学化学化工学院＼cjkstart河南省精细化学品绿色制造协同创新中心 新乡 453007)
}

\begin{abstract}
摘要 以 5-甲酰基嘧啶核甘为原料, 先经与四澳化碳缩合得 5-(2,2-二溴乙烯基)嘧啶核甘类似物, 然后在亚磷酸二乙酯 和三乙胺的作用下发生立体选择性脱溴反应, 高效合成了澳呋啶及其类似物.

关键词 嗅呋啶; 偕二溴代烯烃; 脱澳反应
\end{abstract}

\section{New Synthesis of Brivudine and Its Analogs}
Li, Peiyuan
Zhang, Jianrui
Guo, Shenghai
Zhang, Xinying*
Fan, Xuesen*
(School of Chemistry and Chemical Engineering, Collaborative Innovation Centre of Henan Province for Green Manufacturing of Fine Chemicals, Henan Normal University, Xinxiang 453007)

\begin{abstract}
In this paper, a simple and practical method for the preparation of brivudine (BVDU) and its analog nucleoside derivatives via condensation of the easily obtainable 5 -formyl pyrimidine nucleosides with carbon tetrabromide followed by an efficient and stereoselective debromination promoted by diethyl phosphite and triethylamine is presented.

Keywords brivudine; gem-dibromoalkenes; debromination
\end{abstract}

核苷类药物在病毒性疾病及癌症的治疗方面起到 了很大的作用 ${ }^{[1,2]}$. 其中, 5-[(E)-2-溴乙烯基]-2'-脱氧尿嘧 啶核苷(溴呋啶, brivudine)及其类似物可在病毒胸苷激 酶的催化下进行系列磷酸化, 形成溴呋啶三磷酸酯. 溴 呋啶三磷酸酯可通过抑制病毒 DNA 聚合酶的活性, 进 而起到阻止病毒复制的效果. 澳呋啶是迄今为止所发现 的抗单纯疮疹病毒(HSV-1)及水痘-带状疮疹病毒(VZV) 活性最强的嘧啶核苷衍生物之一, 临床主要用于 HSV-1 和 VZV 引起的皮肤及粘膜感染 ${ }^{[3]}$. 目前, 澳呋啶的合成 方法主要有以下几种: (1)经由预先制备的 5-位官能团化 的尿嘧啶与经活化的呋喃糖基发生偶联反应得到澳呋 啶，其制备过程涉及到高活性偶联试剂的使用、 $\alpha, \beta$ 异构 体的分离以及羟基的保护和脱保护等 ${ }^{[4]}$, 该方法由于存 在步骤多、中间体和产物分离纯化困难以及催化剂和偶 联试剂价格昂贵等问题, 无法实现大量生产; (2)以 5-卤 代-2'-脱氧尿苷为原料, 经钯催化下与丙烯酸酯的偶联 反应及偶联产物的碱性水解得 5-[(E)-2-羒基乙烯基]-2'脱氧尿苷中间体, 该中间体在 $N$-溴代丁二酰亚胺作用 下发生脱羧、澳化反应得到澳呋啶 ${ }^{[5]}$, 该方法克服了第
一种合成方法路线圥长的缺点, 但仍存在原料及金属钯 催化剂价格高、产物分离纯化困难等问题; (3)以 2'-脱氧 尿嘧啶核苷为起始原料, 先后经过羟甲基化、选择性氧 化、Knoevenagel 缩合和 Hunsdiecker 反应合成溴呋啶 ${ }^{[6]}$. 相比较而言, 第三种方法所用试剂价格便宜、效率高, 是一种有着潜在应用前景的新方法. 尽管已有上述现成 的合成溴呋啶的方法, 但由于该类化合物的重要性, 所 以仍有必要发展更加有效且简便、通用的合成新方法.

近年来，偕二卤代烯烃作为一类易制备并具有较高 反应活性的中间体，在多种化合物的合成中得到了广泛 的应用 ${ }^{[7]}$. 其中, Hirao ${ }^{[8]}$ 和 Hayes 等 ${ }^{[86]}$ 发现, 偕二溴代 烯烃可在亚磷酸酯及碱的共同作用下发生脱溴反应，生 成相应的单溴代烯烃, 产物收率高并具有良好的 $E$-选 择性. 最近, 我们通过 5-甲酰基嘧啶核苷与四溴化碳的 缩合反应得到了一系列 5-(2,2-二溴乙烯基)嘧啶核苷类 似物，并以该类化合物为中间体合成了具有重要应用价 值的 6-澳代呋喃[2,3- $d$ 嘧啶双环核甘 ${ }^{[9]}$. 在上述研究成 果的基础上，我们设计了一条合成澳呋啶及其类似物的 新方法, 即以 5-甲酰基嘧啶核苷(1, 图 1)为原料, 先经

*E-mail: xuesen.fan@htu.cn; xinyingzhang@htu.cn

Received October 24, 2014; revised November 22, 2014; published online December 9, 2014.

Project supported by the National Natural Science Foundation of China (Nos. 21172057, 21272058, 21202040), and the Research Fund for the Doctoral Program of Higher Education (No. 20114104110005).

国家自然科学基金(Nos. 21172057, 21272058, 21202040)及教育部博士点基金(No. 20114104110005)资助项目. 
与四溴化碳缩合得到相应的 5-(2,2-二澳乙烯基)嘧啶核 苷(2), 然后经由偕二溴烯烃结构单元的立体选择性脱 溴反应得到 5-[(E)-2-澳乙烯基]嘧啶核苷衍生物 3 .<smiles>[R]n1cc(C=C(Br)Br)c(=O)[nH]c1=O</smiles>

图式 1 (E)-5-(2-溴乙烯基)嘧啶核苷衍生物 3 的设计合成路线 Scheme 1 Proposed synthetic pathway toward (E)-5-(2-bromovinyl)pyrimidine nucleosides (3)

\section{1 结果与讨论}

首先，利用经改进的文献方法 ${ }^{[9,10]}$ 合成了一系列 5-(2,2-二溴乙烯基)嘧啶核苷(2), 结果见表 1 .

随后, 对 2a 的脱溴反应进行了考察. 研究发现, 以 2 equiv. 的亚磷酸二甲酯为还原剂、2 equiv. 的三乙胺为 碱、 $\mathrm{DMF}$ 为介质, 在 $70{ }^{\circ} \mathrm{C}$ 的温度条件下摚拌反应 $6 \mathrm{~h}$, 可以 $69 \%$ 的收率得到目标产物 $(E)-5$-(2-溴乙烯基)-2'-脱 氧-3',5'-双乙酰尿嘧啶核苷(3a，表 2, Entry 1). 当以亚磷 酸二乙酯为还原剂时, 产物收率可提高到 $72 \%$ (表 2, Entry 2). 以亚磷酸二乙酯为还原剂, 考察了不同介质对 反应的影响. 研究发现, 当反应在 $N, N$-二甲基甲酰胺 (DMF) 进行时效果最好, 以二氯甲烷或乙醇为溶剂时, 仅有少量的产物生成(表 2, Entries 3,4). 随后又探讨了 不同温度对反应的影响, 结果表明, 当反应在室温或 $50{ }^{\circ} \mathrm{C}$ 进行时, 产物收率下降(表 2, Entries 5, 6); 而将反 应温度从 $70{ }^{\circ} \mathrm{C}$ 提高到 $90{ }^{\circ} \mathrm{C}$ 或 $120{ }^{\circ} \mathrm{C}$ 时, 产物收率也 没有改善(表 2, Entries 7,8). 综上, 生成 3a 的较佳反应
条件为: 亚磷酸二乙酯 (2 equiv.) 为还原剂、三乙胺 (2 equiv.) 为碱、 $\mathrm{DMF}$ 为溶剂、反应温度为 $70{ }^{\circ} \mathrm{C}$ 、反应时 间为 $6 \mathrm{~h}$, 在该条件下, $3 \mathrm{a}$ 的收率为 $72 \%$ (表 2, Entry 2). 需要指出的是, 上述反应之所以表现出良好的 $E$-选择 性，是因为发生消除反应的关键中间体中两个较大的取 代基团(嘧啶环和澳原子)处于反位时相对更稳定，从而 使反应主要朝着生成 $E$-型产物的方向进行 ${ }^{[8]}$.

利用表 2 中的最佳反应条件，我们又尝试了一系列 5-(2,2-二溴乙烯基)嘧啶核苷(2)的脱溴反应，结果见表 3. 表 3 中的数据表明, 不同种类的 2,2-二溴乙烯基取代 嘧啶核苷(2)均能在亚磷酸二乙酯和三乙胺的作用下发 生预期的立体选择性脱溴反应，生成相应的 $(E)-5-(2-$ 溴 乙烯基)嘧啶核苷衍生物 3.

进一步的研究发现, (E)-5-(2-澳乙烯基)嘧啶核苷衍 生物 3 可在二丁基氧化锡的作用下脱除相应的乙酰保护 基 $^{[11]}$, 从而得到溴呋啶 $4 a$ 及其类似物 $4 b \sim 4 c$. 该反应 操作简单, 条件温和, 具体结果见表 4 .

在随后的研究中, 我们又尝试了以 $\mathbf{3 a}$ 为底物合成溴呋 啶的胞嘧啶核苷类似物. 首先, 以 $3 \mathrm{a}$ 为起始原料, 在 Lawesson 试剂的作用下得到(E)-5-(2-澳乙烯基)-4-硫代 -2'-脱氧-3',5'-双乙酰尿嘧啶核苷(5, 图 2), 然后通过硫 甲基化得到关键中间体 (E)-5-(2-溴乙烯基)-4-甲硫基 $2^{\prime}$-脱氧-3',5'-双乙酰嘧啶核苷 (6). 在异丙醇介质中, 分 别以氨水和甲胺水溶液为氨基化试剂, 可以较高的收率 得到( $E$ )-5-(2-溴乙烯基)-2'-脱氧胞嘧啶核苷(7)及其 $N$-甲 基化衍生物 8. 值得注意的是，在上述反应中氨水和甲 氨水溶液不仅是有效的氨基化试剂, 而且可以作为脱保 护试剂高效脱除底物中的乙酰基，直接得到最终产物.

表 1 5-(2,2-二溴乙烯基)嘧啶核苷衍生物(2)的制备 ${ }^{a}$

Table 1 Preparation of 5-(2,2-dibromovinyl)pyrimidine nucleosides (2)

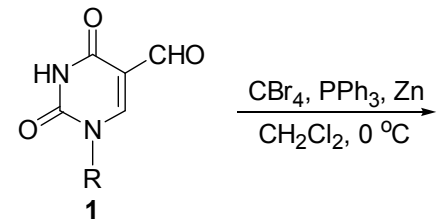<smiles>[R]n1cc(C=C(Br)Br)c(=O)[nH]c1=O</smiles>

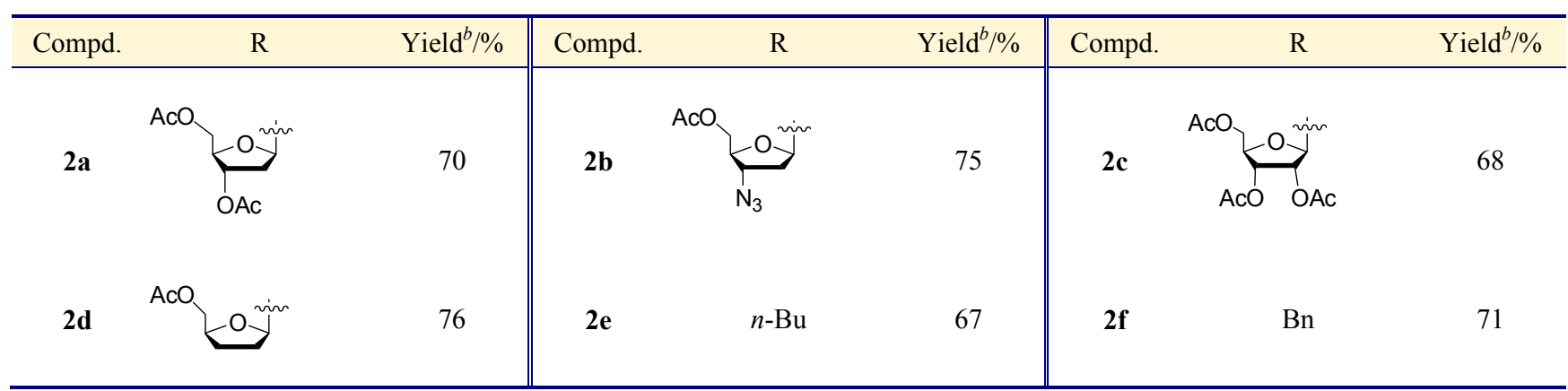

${ }^{a}$ Reaction conditions: $2 \mathrm{mmol}$ of $1,6 \mathrm{mmol}$ of $\mathrm{PPh}_{3}, 6 \mathrm{mmol}$ of zinc powder, $6 \mathrm{mmol}$ of $\mathrm{CBr}_{4}{ }^{b}$ Isolated yield. 
表 2 合成 $3 \mathbf{a}$ 的反应条件优化研究 ${ }^{a}$

Table 2 Optimization study on the preparation of $\mathbf{3 a}$<smiles>CC(=O)OCC1OC2CC(n3cc(C=C(Br)Br)c(=O)[nH]c3=O)C1OC2=O</smiles>

2a

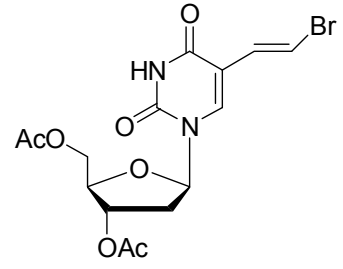

$3 a$

\begin{tabular}{|c|c|c|c|c|c|c|}
\hline Entry & Base & Solvent & Phosphite & $T /{ }^{\circ} \mathrm{C}$ & $t / \mathrm{h}$ & Yield $^{b} / \%$ \\
\hline 1 & $\mathrm{Et}_{3} \mathrm{~N}$ & DMF & $(\mathrm{MeO})_{2} \mathrm{P}(\mathrm{O}) \mathrm{H}$ & 70 & 6 & 69 \\
\hline 2 & $\mathrm{Et}_{3} \mathrm{~N}$ & DMF & $(\mathrm{EtO})_{2} \mathrm{P}(\mathrm{O}) \mathrm{H}$ & 70 & 6 & 72 \\
\hline 3 & $\mathrm{Et}_{3} \mathrm{~N}$ & $\mathrm{CH}_{2} \mathrm{Cl}_{2}$ & $(\mathrm{EtO})_{2} \mathrm{P}(\mathrm{O}) \mathrm{H}$ & 70 & 6 & Trace \\
\hline 4 & $\mathrm{Et}_{3} \mathrm{~N}$ & $\mathrm{EtOH}$ & $(\mathrm{EtO})_{2} \mathrm{P}(\mathrm{O}) \mathrm{H}$ & 70 & 6 & Trace \\
\hline 5 & $\mathrm{Et}_{3} \mathrm{~N}$ & DMF & $(\mathrm{EtO})_{2} \mathrm{P}(\mathrm{O}) \mathrm{H}$ & r.t. & 10 & 32 \\
\hline 6 & $\mathrm{Et}_{3} \mathrm{~N}$ & DMF & $(\mathrm{EtO})_{2} \mathrm{P}(\mathrm{O}) \mathrm{H}$ & 50 & 10 & 51 \\
\hline 7 & $\mathrm{Et}_{3} \mathrm{~N}$ & DMF & $(\mathrm{EtO})_{2} \mathrm{P}(\mathrm{O}) \mathrm{H}$ & 90 & 6 & 71 \\
\hline 8 & $\mathrm{Et}_{3} \mathrm{~N}$ & DMF & $(\mathrm{EtO})_{2} \mathrm{P}(\mathrm{O}) \mathrm{H}$ & 120 & 6 & 63 \\
\hline
\end{tabular}

${ }^{a}$ The reactions were run with $0.5 \mathrm{mmol}$ of $\mathbf{2 a}, 1 \mathrm{mmol}$ of $(\mathrm{EtO})_{2} \mathrm{P}(\mathrm{O}) \mathrm{H}$ or $(\mathrm{MeO})_{2} \mathrm{P}(\mathrm{O}) \mathrm{H}, 1 \mathrm{mmol} \mathrm{of} \mathrm{Et}_{3} \mathrm{~N} .{ }^{b}$ Isolated yield.

表 3 (E)-5-(2-溴乙烯基)嘧啶核苷衍生物 3 的制备 ${ }^{a}$

Table 3 Preparation of (E)-5-(2-bromovinyl)pyrimidine nucleosides (3)

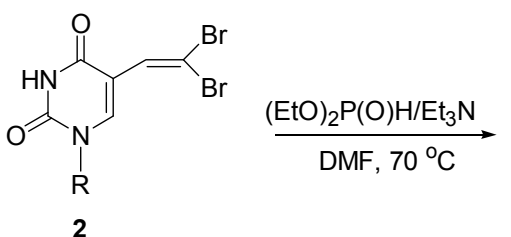<smiles>[R]n1cc(/C=C/Br)c(=O)[nH]c1=O</smiles>

3

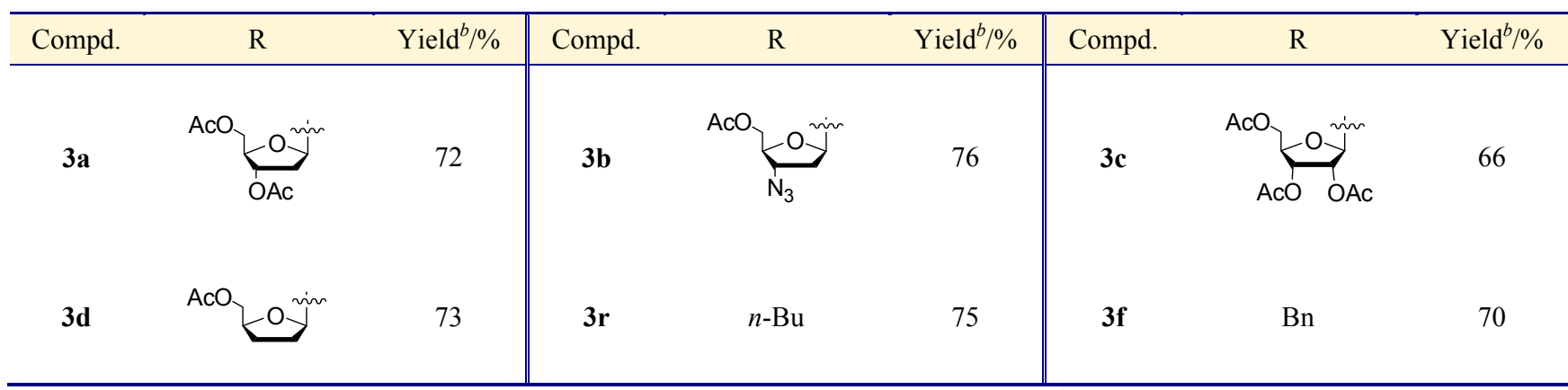

${ }^{a}$ The reactions were run with $1 \mathrm{mmol}$ of $\mathbf{2}, 2 \mathrm{mmol}$ of $(\mathrm{EtO})_{2} \mathrm{P}(\mathrm{O}) \mathrm{H}, 2 \mathrm{mmol}$ of $\mathrm{Et}_{3} \mathrm{~N}$ in $\mathrm{DMF}$ at $70{ }^{\circ} \mathrm{C}$ for $6 \mathrm{~h}$. ${ }^{\mathrm{b}}$ Isolated yield.

\section{2 结论}

本文以易得的 5-甲酰基嘧啶核苷(1)为原料, 先经 与四溴化碳缩合得 5-(2,2-二溴乙烯基)嘧啶核苷(2), 然 后在亚磷酸二乙酯及三乙胺的作用下发生立体选择性 脱溴反应, 生成 5-[(E)-2-澳乙烯基]嘧啶核苷(3), 最后经 乙酰基脱除即可得到溴呋啶及其类似物 4 . 另外, 以 $3 \mathrm{a}$ 为原料, 经硫甲基化和氨解, 方便、高效地合成了溴呋 啶的胞嘧啶核苷衍生物 7, 8. 与文献方法相比, 本方法 的合成路线较短、无需使用昂贵的钯催化剂, 反应条件 相对温和，而且避免了文献方法中不易控制的溴化反应
等步骤, 从而为溴呋啶及其类似物的合成提供了一条经 济、实用、有效的新途径.

\section{3 实验部分}

\section{1 仪器与试剂}

5-(2,2-二澳乙烯基)嘧啶核苷(2)的合成见文献 [9, 10]. ${ }^{1} \mathrm{H}$ NMR 及 ${ }^{13} \mathrm{C}$ NMR 使用 Bruker AC-400 核磁共振 仪测定, TMS 为内标, $\mathrm{CDCl}_{3} 、 \mathrm{DMSO}-d_{6} 、 \mathrm{CD}_{3} \mathrm{OD}$ 或 $\left(\mathrm{CD}_{3}\right)_{2} \mathrm{CO}$ 为溶剂; 高分辨质谱测定使用 Bruker micrOTOF 质谱仪. 
表 4 (E)-5-(2-溴乙烯基)嘧啶核苷衍生物(3)的脱乙酰化 ${ }^{a}$

Table 4 Deacetylation of (E)-5-(2-bromovinyl)pyrimidine nucleosides (3)
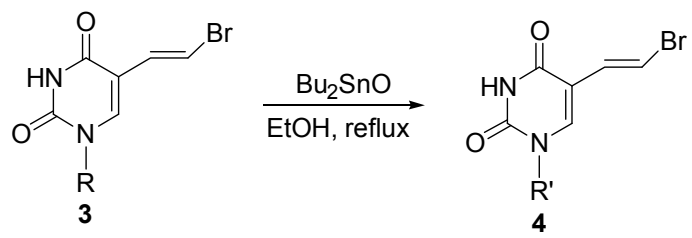

\begin{tabular}{|c|c|c|c|c|c|c|c|c|c|c|c|}
\hline Compd. & $\mathrm{R}$ & Yield $^{b} / \%$ & Compd. & $\mathrm{R}$ & Yield $^{b} / \%$ & Compd. & $\mathrm{R}$ & Yield $^{b} / \%$ & Compd. & $\mathrm{R}$ & Yield $^{b} / \%$ \\
\hline $4 a$ & ${ }_{\mathrm{O}}$ & 80 & $4 b$ & $\mathrm{~N}_{3}$ & 85 & $4 c$ & $\mathrm{OH}$ & 78 & 4d & & 86 \\
\hline
\end{tabular}

${ }^{a}$ Reaction conditions: $0.5 \mathrm{mmol}$ of 3, $0.5 \mathrm{mmol}$ of $\mathrm{Bu}_{2} \mathrm{SnO}, 10 \mathrm{~mL}$ of EtOH, reflux. ${ }^{b}$ Isolated yield.

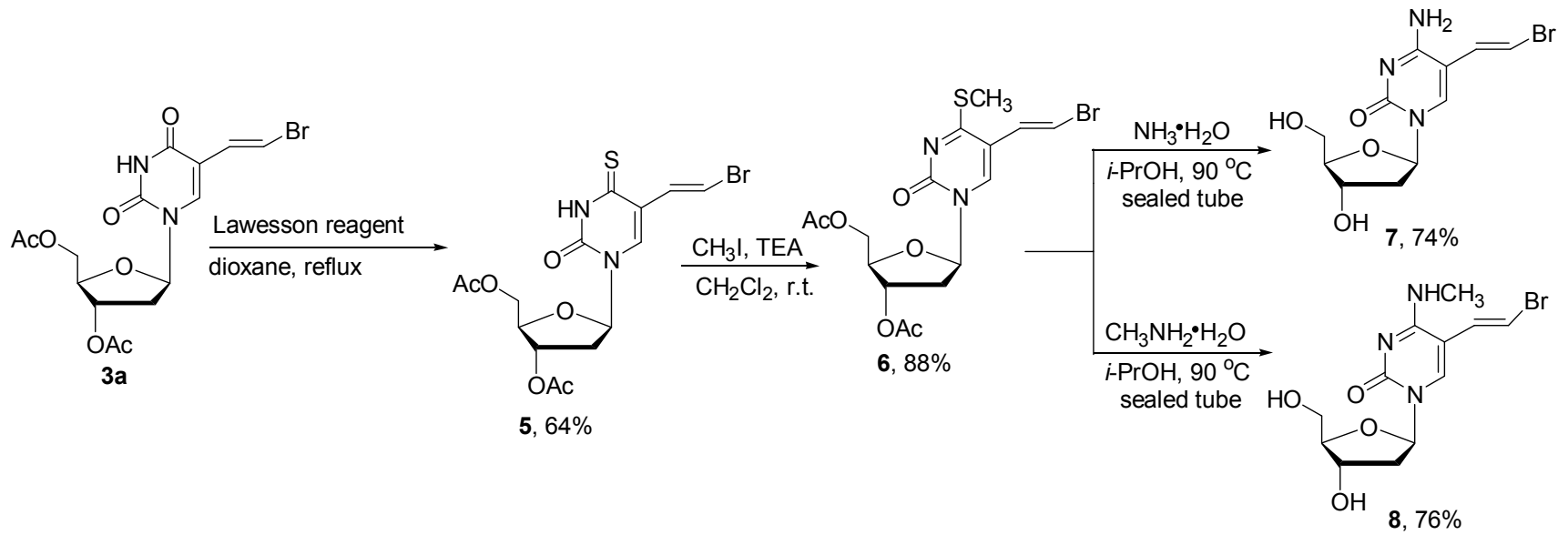

图式 2 (E)-5-(2-溴乙烯基)-2'-脱氧胞嘧啶核苷(7)及其 $N$-甲基化衍生物 8 的合成

Scheme 2 Synthesis of E-5-(2-bromovinyl)-2'-deoxycytidine (7) and its $N$-methylation analog (8)

\section{2 实验方法}

3.2.1 (E)-5-(2-溴乙烯基)-2'-脱氧-3',5'-双乙酰尿嘧啶 核苷(3a) 的合成

将 $2 \mathbf{a}(1 \mathrm{mmol})$ 加入到反应瓶中并用 DMF (5 mL) 溶 解, 再依次加入亚磷酸二乙酯 $(2 \mathrm{mmol})$ 和三乙胺 $(2$ $\mathrm{mmol})$. 将反应瓶置于 $70{ }^{\circ} \mathrm{C}$ 油浴中搅拌反应, 待 $\mathrm{TLC}$ 检测反应完全后, 加入适量水, 再用乙酸乙酯萃取(10 $\mathrm{mL} \times 3)$, 合并有机相, 用饱和食盐水洗涤 2 次, 再用无 水硫酸钠干燥, 旋蒸浓缩, 残留物用石油醚/乙酸乙酯 $(V: V=1: 1)$ 作洗脱剂进行柱层析纯化得 $\mathbf{3 a}$.

(E)-5-(2-溴乙烯基)-2'-脱氧-3',5'-双乙酰尿嘧啶核苷 (3a): ${ }^{1} \mathrm{H}$ NMR (400 MHz, $\left.\mathrm{CDCl}_{3}\right) \delta: 2.08 \sim 2.16(\mathrm{~m}, 7 \mathrm{H}$, $\left.\mathrm{CH}_{3} \times 2, \mathrm{H} 2^{\prime}-1\right), 2.54 \sim 2.59\left(\mathrm{~m}, 1 \mathrm{H}, \mathrm{H} 2^{\prime}-2\right), 4.29 \sim 4.44$ $\left(\mathrm{m}, 3 \mathrm{H}, \mathrm{H} 3^{\prime}, \mathrm{H} 5^{\prime}\right), 5.20 \sim 5.21\left(\mathrm{~m}, 1 \mathrm{H}, \mathrm{H} 4^{\prime}\right), 6.27(\mathrm{t}, J=8.0$ $\mathrm{Hz}, 1 \mathrm{H}, \mathrm{H1}$ ), 6.67 (d, $J=14.0 \mathrm{~Hz}, 1 \mathrm{H}$, vinyl-H1), 7.41 (d, $J=14.0 \mathrm{~Hz}, 1 \mathrm{H}$, vinyl-H2), 7.50 (s, 1H, H6), 9.27 (br s, $1 \mathrm{H}, \mathrm{NH}) ;{ }^{13} \mathrm{C}$ NMR (100 MHz, $\left.\mathrm{CDCl}_{3}\right) \delta: 20.81,20.84$, $37.9,63.7,74.0,82.6,85.6,110.1,111.7,128.2$, 136.7,
149.4, 161.6, 170.3, 170.4. HRMS calcd for $\mathrm{C}_{15} \mathrm{H}_{17} \mathrm{Br}-$ $\mathrm{N}_{2} \mathrm{O}_{7} \mathrm{Na}[\mathrm{M}+\mathrm{Na}]^{+}: 439.0117$, found 439.0128 .

(E)-5-(2-溴乙烯基)-2'-脱氧-3'-叠氮-5'-乙酰尿嘧啶 核苷(3b): 其制备过程同 3a. ${ }^{1} \mathrm{H}$ NMR (400 $\left.\mathrm{MHz}, \mathrm{CDCl}_{3}\right)$ $\delta: 2.15\left(\mathrm{~s}, 3 \mathrm{H}, \mathrm{CH}_{3}\right), 2.39 \sim 2.42\left(\mathrm{~m}, 1 \mathrm{H}, \mathrm{H} 2^{\prime}-1\right), 2.55 \sim$ 2.58 (m, 1H, H2'-2), $4.12 \sim 4.13\left(\mathrm{~m}, 1 \mathrm{H}, \mathrm{H} 3{ }^{\prime}\right), 4.18 \sim 4.20$ $\left(\mathrm{m}, 1 \mathrm{H}, \mathrm{H}^{\prime}\right), 4.30 \sim 4.34\left(\mathrm{~m}, 1 \mathrm{H}, \mathrm{H} 4^{\prime}\right), 4.39 \sim 4.44(\mathrm{~m}, 1 \mathrm{H}$, H5'), 6.08 (t, $\left.J=6.0 \mathrm{~Hz}, 1 \mathrm{H}, \mathrm{H} 1^{\prime}\right), 6.65$ (d, $J=13.6 \mathrm{~Hz}$, 1H, vinyl-H1), 7.40 (d, $J=13.6 \mathrm{~Hz}, 1 \mathrm{H}$, vinyl-H2), 7.46 (s, 1H, H6), 9.48 (br s, $1 \mathrm{H}, \mathrm{NH}) ;{ }^{13} \mathrm{C} \mathrm{NMR}(100 \mathrm{MHz}$, $\left.\mathrm{CDCl}_{3}\right) \delta: 20.8,38.0,60.2,63.2,82.2,86.2,110.3,111.5$, 128.1, 137.2, 149.1, 161.6, 170.4. HRMS calcd for $\mathrm{C}_{13} \mathrm{H}_{14} \mathrm{BrN}_{5} \mathrm{O}_{5} \mathrm{Na}[\mathrm{M}+\mathrm{Na}]^{+}:$422.0076, found 422.0085 .

(E)-5-(2-溴乙烯基)-2',3',5'-三乙酰尿嘧啶核苷(3c): 其制备过程同 3a. ${ }^{1} \mathrm{H}$ NMR (400 MHz, $\left.\mathrm{CDCl}_{3}\right) \delta: 2.09$ (s, $\left.3 \mathrm{H}, \mathrm{CH}_{3}\right), 2.12\left(\mathrm{~s}, 3 \mathrm{H}, \mathrm{CH}_{3}\right), 2.16\left(\mathrm{~s}, 3 \mathrm{H}, \mathrm{CH}_{3}\right), 4.32 \sim 4.42$ (m, 3H, H3', H5'), 5.32 5.35 (m, 2H, H2', H4'), 6.03 (d, $\left.J=4.4 \mathrm{~Hz}, 1 \mathrm{H}, \mathrm{H} 1^{\prime}\right), 6.64$ (d, $J=13.6 \mathrm{~Hz}, 1 \mathrm{H}$, vinyl-H1), 
7.38 (s, 1H, H6), 7.40 (d, $J=11.6 \mathrm{~Hz}, 1 \mathrm{H}$, vinyl-H2), 9.35 (br s, $1 \mathrm{H}, \mathrm{NH}) ;{ }^{13} \mathrm{C}$ NMR $\left(100 \mathrm{MHz}, \mathrm{CDCl}_{3}\right) \delta: 20.3,20.4$, 20.7, 63.0, 70.0, 72.8, 79.9, 87.6, 110.5, 112.1, 128.1, 137.0, 149.3, 161.4, 169.69, 169.72, 170.3. HRMS calcd for $\mathrm{C}_{17} \mathrm{H}_{19} \mathrm{BrN}_{2} \mathrm{O}_{9} \mathrm{Na}[\mathrm{M}+\mathrm{Na}]^{+}$: 497.0172, found 497.0186.

(E)-5-(2-澳乙烯基)-2', 3'-双脱氧-5'-乙酰尿嘧啶核苷 (3d): 其制备过程同 3a. ${ }^{1} \mathrm{H}$ NMR $\left(400 \mathrm{MHz}, \mathrm{CDCl}_{3}\right) \delta$ : $1.74 \sim 1.79\left(\mathrm{~m}, 1 \mathrm{H}, \mathrm{H} 3^{\prime}-1\right), 2.03 \sim 2.11\left(\mathrm{~m}, 2 \mathrm{H}, \mathrm{H} 2^{\prime}-1\right.$, H3'-2), $2.13\left(\mathrm{~s}, 1 \mathrm{H}, \mathrm{CH}_{3}\right), 2.43 \sim 2.49\left(\mathrm{~m}, 1 \mathrm{H}, \mathrm{H} 2^{\prime}-2\right)$, $\left.4.27 \sim 4.43\left(\mathrm{~m}, 3 \mathrm{H}, \mathrm{H} 4{ }^{\prime}, \mathrm{H} 5\right)^{\prime}\right), 6.01$ (q, $J=6.4 \mathrm{~Hz}, 1 \mathrm{H}$, H1'), 6.69 (d, $J=13.2 \mathrm{~Hz}, 1 \mathrm{H}$, vinyl-H1), 7.39 (d, $J=13.2$ Hz, 1H, vinyl-H2), 7.66 (s, 1H, H6), 9.84 (br s, 1H, NH); ${ }^{13} \mathrm{C}$ NMR $\left(100 \mathrm{MHz}, \mathrm{CDCl}_{3}\right) \delta: 20.9,25.3,32.8,64.6$, 79.4, 87.0, 109.8, 110.7, 128.4, 137.5, 149.3, 161.7, 170.6. HRMS calcd for $\mathrm{C}_{13} \mathrm{H}_{15} \mathrm{BrN}_{2} \mathrm{O}_{5} \mathrm{Na}[\mathrm{M}+\mathrm{Na}]^{+}: 381.0062$, found 381.0071 .

1-正丁基-(E)-5-(2-溴乙烯基)-嘧啶-2,4(1H,3H)-二 酩 (3e): 其制备过程同 3a. ${ }^{1} \mathrm{H}$ NMR (400 $\mathrm{MHz}$, DMSO- $\left.d_{6}\right) \delta: 0.88\left(\mathrm{t}, J=7.2 \mathrm{~Hz}, 3 \mathrm{H}, \mathrm{CH}_{3}\right), 1.23 \sim 1.30(\mathrm{~m}$, $\left.2 \mathrm{H}, \mathrm{CH}_{2}\right), 1.53 \sim 1.60\left(\mathrm{~m}, 2 \mathrm{H}, \mathrm{CH}_{2}\right), 3.66(\mathrm{t}, J=7.2 \mathrm{~Hz}$, $\left.2 \mathrm{H}, \mathrm{CH}_{2}\right), 6.79$ (d, $J=13.6 \mathrm{~Hz}, 1 \mathrm{H}$, vinyl-H1), 7.24 (d, $J=$ $14.0 \mathrm{~Hz}, 1 \mathrm{H}$, vinyl-H2), 11.53 (br s, $1 \mathrm{H}, \mathrm{NH}$ ); ${ }^{13} \mathrm{C}$ NMR $\left(100 \mathrm{MHz}, \mathrm{DMSO}-d_{6}\right) \delta: 13.9,19.5,30.8,48.0,106.5$, $109.5,130.0,145.1,150.2$, 162.5. HRMS calcd for $\mathrm{C}_{10} \mathrm{H}_{13} \mathrm{BrN}_{2} \mathrm{O}_{2} \mathrm{Na}[\mathrm{M}+\mathrm{Na}]^{+}: 295.0058$, found 295.0052 .

1-芐基-(E)-5-(2-溴乙烯基)-嘧啶-2,4(1H,3H)-二酮 (3f): 其制备过程同 3a. ${ }^{1} \mathrm{H}$ NMR (400 $\left.\mathrm{MHz}, \mathrm{CDCl}_{3}\right) \delta$ : $4.94\left(\mathrm{~s}, 2 \mathrm{H}, \mathrm{CH}_{2}\right), 6.58$ (d, $J=13.6 \mathrm{~Hz}, 1 \mathrm{H}$, vinyl-H1), 7.10 (s, 1H, H6), 7.26 7.42 (m, 6H, vinyl-H2, Ph-H), 9.07 (br s, $1 \mathrm{H}, \mathrm{NH}) ;{ }^{13} \mathrm{C} \mathrm{NMR}\left(100 \mathrm{MHz}, \mathrm{CDCl}_{3}\right) \delta: 51.4$, 110.3, 111.7, 127.9, 128.1(重碳), 128.8, 129.2(重碳), 134.7, 141.4, 150.0, 161.7. HRMS calcd for $\mathrm{C}_{13} \mathrm{H}_{11} \mathrm{BrN}_{2} \mathrm{O}_{2} \mathrm{Na}[\mathrm{M}+\mathrm{Na}]^{+}: 328.9902$, found 328.9918.

\subsection{2 (E)-5-(2-溴乙烯基)-2'-脱氧尿嘧啶核苷 (4a) 的} 合成

将 $3 \mathbf{a}(0.5 \mathrm{mmol})$ 和乙醇 $(10 \mathrm{~mL})$ 加入到反应瓶中, 在摚拌下加入 $\mathrm{Bu}_{2} \mathrm{SnO}(0.5 \mathrm{mmol})$, 然后加热回流反应 6 $\mathrm{h}$, 待 TLC 检测反应完全后, 减压浓缩, 残留物用二氯 甲烷/甲醇 $(V: V=20: 1)$ 作洗脱剂进行柱层析分离得 4a. ${ }^{1} \mathrm{H}$ NMR (400 MHz, DMSO- $\left.d_{6}\right) \delta: 2.09 \sim 2.12(\mathrm{~m}, 2 \mathrm{H}$, H2'), 3.52 3.62 (m, 2H, H5'), 3.75 (d, $J=3.2 \mathrm{~Hz}, 1 \mathrm{H}$, H5'-OH), 4.22 (t, $\left.J=3.6 \mathrm{~Hz}, 1 \mathrm{H}, \mathrm{H} 3^{\prime}-\mathrm{OH}\right), 5.06$ (t, $J=5.2$ Hz, 1H, H4'), 5.22 (d, $J=3.6 \mathrm{~Hz}, 1 \mathrm{H}, \mathrm{H} 3$ '), 6.10 (t, $J=6.8$ $\left.\mathrm{Hz}, 1 \mathrm{H}, \mathrm{H1} 1^{\prime}\right), 6.81$ (d, $J=13.6 \mathrm{~Hz}, 1 \mathrm{H}$, vinyl-H1), 7.21 (d,
$J=13.6 \mathrm{~Hz}, 1 \mathrm{H}$, vinyl-H2), 8.05 (s, 1H, H6), 11.53 (br s, $1 \mathrm{H}, \mathrm{NH}) ;{ }^{13} \mathrm{C}$ NMR (100 MHz, DMSO- $\left.d_{6}\right) \delta: 40.9,61.4$, 70.3, 84.9, 87.9, 106.9, 110.1, 130.3, 139.9, 149.7, 162.1. HRMS calcd for $\mathrm{C}_{11} \mathrm{H}_{13} \mathrm{BrN}_{2} \mathrm{O}_{5} \mathrm{Na}[\mathrm{M}+\mathrm{Na}]^{+}: 354.9906$, found 354.9908 .

(E)-5-(2-溴乙烯基)-2'-脱氧-3'-叠氮尿嘧啶核苷 (4b): 制备过程与 4a 类似, 反应时间为 $2 \mathrm{~h} ;{ }^{1} \mathrm{H}$ NMR (400 MHz, DMSO- $d_{6}$ ) $\delta: 2.31 \sim 2.47\left(\mathrm{~m}, 2 \mathrm{H}, \mathrm{H} 2^{\prime}\right), 3.60 \sim 3.72$ (m, 2H, H5'), 3.84 (d, J=4.8 Hz, 1H, H5'-OH), 4.42 (q, $\left.J=6.0 \mathrm{~Hz}, 1 \mathrm{H}, \mathrm{H} 3^{\prime}\right), 5.30$ (t, $\left.J=4.8 \mathrm{~Hz}, 1 \mathrm{H}, \mathrm{H} 4{ }^{\prime}\right), 6.07$ (t, $J=6.0 \mathrm{~Hz}, 1 \mathrm{H}, \mathrm{H1}$ ), $6.85(\mathrm{~d}, J=13.2 \mathrm{~Hz}, 1 \mathrm{H}$, vinyl-H1), 7.48 (d, 13.2 Hz, 1H, vinyl-H2), 8.06 (s, 1H, H6), 11.61 (br s, $1 \mathrm{H}, \mathrm{NH}) ;{ }^{13} \mathrm{C}$ NMR $\left(100 \mathrm{MHz}\right.$, DMSO- $\left.d_{6}\right) \delta: 37.1$, 59.8, 60.8, 84.5, 84.8, 107.0, 110.1, 130.2, 139.8, 149.7, 162.1. HRMS calcd for $\mathrm{C}_{11} \mathrm{H}_{12} \mathrm{BrN}_{5} \mathrm{O}_{4} \mathrm{Na}[\mathrm{M}+\mathrm{Na}]^{+}$: 379.9971, found 379.9988 .

(E)-5-(2-溴乙烯基)-尿嘧啶核苷(4c)：制备过程与 4a 类似, 反应时间为 $48 \mathrm{~h} .{ }^{1} \mathrm{H}$ NMR (400 MHz, $\left.\mathrm{CD}_{3} \mathrm{OD}\right) \delta$ : $3.74 \sim 3.91\left(\mathrm{~m}, 2 \mathrm{H}, \mathrm{H} 2^{\prime}, \mathrm{H} 4^{\prime}\right), 4.00 \sim 4.02\left(\mathrm{~m}, 1 \mathrm{H}, \mathrm{H} 3^{\prime}\right)$, $4.15 \sim 4.17$ (m, 2H, H5'), 5.88 (t, $\left.J=6.0 \mathrm{~Hz}, 1 \mathrm{H}, \mathrm{H1} 1^{\prime}\right), 6.77$ (d, $J=13.6 \mathrm{~Hz}, 1 \mathrm{H}$, vinyl-H1), 7.28 (d, $13.6 \mathrm{~Hz}, 1 \mathrm{H}$, vinyl-H2), 8.21 (s, 1H, H6); ${ }^{13} \mathrm{C}$ NMR (100 MHz, $\mathrm{CD}_{3} \mathrm{OD}$ ) $\delta: 60.3,69.3,74.6,84.7,89.5,107.2,110.5,128.8,138.9$, 150.0, 162.3. HRMS calcd for $\mathrm{C}_{11} \mathrm{H}_{13} \mathrm{BrN}_{2} \mathrm{O}_{6} \mathrm{Na}[\mathrm{M}+$ $\mathrm{Na}^{+}: 370.9855$, found 370.9864 .

(E)-5-(2-溴乙烯基)-2',3'-双脱氧尿嘧啶核苷(4d)：制 备过程与 4a 类似, 反应时间为 $2 \mathrm{~h} .{ }^{1} \mathrm{H}$ NMR $(400 \mathrm{MHz}$, $\left.\left(\mathrm{CD}_{3}\right)_{2} \mathrm{CO}\right) \delta: 1.91 \sim 1.97\left(\mathrm{~m}, 1 \mathrm{H}, \mathrm{H} 3{ }^{\prime}-1\right), 2.04 \sim 2.09(\mathrm{~m}$, $1 \mathrm{H}, \mathrm{H} 2$ '-1), $2.12 \sim 2.19$ (m, 1H, H2'-2), 2.35 2.44 (m, 1H, H3'-2), 3.74 3.78 (m, 1H, H5'-1), 3.95 3.99 (m, 1H, H5'-2), 4.15 4.21 (m, 1H, H4'), 4.52 (s, 1H, H5'-OH), $6.01 \sim 6.04(\mathrm{~m}, 1 \mathrm{H}, \mathrm{H1}), 6.75(\mathrm{~d}, J=13.2 \mathrm{~Hz}, 1 \mathrm{H}$, vinyl-H1), 7.31 (d, $J=13.2 \mathrm{~Hz}, 1 \mathrm{H}$, vinyl-H2), 8.41 (s, $1 \mathrm{H}$, H6), 10.33 (br s, $1 \mathrm{H}, \mathrm{NH}) ;{ }^{13} \mathrm{C}$ NMR $(100 \mathrm{MHz}$, $\left.\left(\mathrm{CD}_{3}\right)_{2} \mathrm{CO}\right) \delta: 23.9,32.8,62.0,82.6,86.3,106.2,109.3$, 130.0, 139.8, 149.3, 161.7. HRMS calcd for $\mathrm{C}_{11} \mathrm{H}_{13} \mathrm{Br}-$ $\mathrm{N}_{2} \mathrm{O}_{4} \mathrm{Na}[\mathrm{M}+\mathrm{Na}]^{+}: 338.9957$, found 338.9942 .

3.2.3 (E)-5-(2-溴乙烯基)-4-硫代-2'-脱氧- $3^{\prime}, 5^{\prime}$ - 双乙 酰尿嘧啶核苷(5)的合成

3a (1 mmol)、二氧六环 $(5 \mathrm{~mL})$ 和 Lawesson 试剂(1.4 $\mathrm{mmol}$ )依次加入到圆底烧瓶中, 摚拌均匀, 然后加热回 流反应. 待 TLC 检测反应完全后, 加入水淬灭反应, 所 得混合物用乙酸乙酯萃取 $(10 \mathrm{~mL} \times 3)$, 合并有机相并用 饱和 $\mathrm{K}_{2} \mathrm{CO}_{3}$ 水溶液洗涤至 $\mathrm{pH}$ 值大于 7. 有机相用无水 $\mathrm{Na}_{2} \mathrm{SO}_{4}$ 干燥, 旋蒸除去溶剂, 粗产品用石油醚/乙酸乙 
酯 $(V: V=2: 1)$ 作洗脱剂进行柱层析分离得产物 $\mathbf{5}$, 收 率 64\%. ${ }^{1} \mathrm{H}$ NMR $\left(400 \mathrm{MHz}, \mathrm{CDCl}_{3}\right) \delta: 2.07\left(\mathrm{~s}, 3 \mathrm{H}, \mathrm{CH}_{3}\right)$, $2.08\left(\mathrm{~s}, 3 \mathrm{H}, \mathrm{CH}_{3}\right), 2.17 \sim 2.24$ (m, $\left.1 \mathrm{H}, \mathrm{H} 2^{\prime}-1\right), 2.56 \sim 2.61$ (m, 1H, H2'-2), 4.29 4.41 (m, 3H, H3', H5'), $5.18 \sim 5.22$ (m, 1H, H4'), 6.20 (t, $J=6.8 \mathrm{~Hz}, 1 \mathrm{H}, \mathrm{H1}$ '), 6.78 (d, $J=13.6$ $\mathrm{Hz}, 1 \mathrm{H}$, vinyl-H1), 7.15 (d, $J=13.6 \mathrm{~Hz}, 1 \mathrm{H}$, vinyl-H2), 7.55 (s, 1H, H6), 10.76 (br s, 1H, NH); ${ }^{13} \mathrm{C}$ NMR (100 $\left.\mathrm{MHz}, \mathrm{CDCl}_{3}\right) \delta: 20.9,21.0,38.2,63.7,73.9,83.0,86.2$, $108.0,120.5,130.9,131.4,147.1,170.4,170.5,187.3$. HRMS calcd for $\mathrm{C}_{15} \mathrm{H}_{17} \mathrm{BrN}_{2} \mathrm{O}_{6} \mathrm{SNa}[\mathrm{M}+\mathrm{Na}]^{+}: 454.9889$, found 454.9912 .

3.2.4 (E)-5-(2-溴乙烯基)-4-硫甲基-2'-脱氧-3',5'-双 乙酰尿嘧啶核苷(6)的合成

将 $5(1 \mathrm{mmol})$ 和二氯甲烷 $(5 \mathrm{~mL})$ 加入到圆底烧瓶中, 搅拌溶解, 然后加入三乙胺 $(4 \mathrm{mmol})$ 和碘甲烷 $(3 \mathrm{mmol})$, 室温摚拌反应, 待 TLC 检测反应结束后, 加入水淬灭反 应, 再加入饱和 $\mathrm{NaCl}$ 水溶液并用二氯甲烷 $(20 \mathrm{~mL} \times 3)$ 萃取, 有机相用无水 $\mathrm{Na}_{2} \mathrm{SO}_{4}$ 干燥, 旋蒸除去溶剂, 粗产 品用石油醚/乙酸乙酯 $(V: V=1: 1)$ 作洗脱剂进行柱层 析分离得产物 6, 收率 $88 \% .{ }^{1} \mathrm{H}$ NMR $\left(400 \mathrm{MHz}, \mathrm{CDCl}_{3}\right)$ $\delta: 2.01 \sim 2.09\left(\mathrm{~m}, 7 \mathrm{H}, \mathrm{CH}_{3} \times 2, \mathrm{H} 2^{\prime}-1\right), 2.50\left(\mathrm{~s}, 3 \mathrm{H}, \mathrm{CH}_{3}\right)$, $2.72 \sim 2.77$ (m, 1H, H2'-2), 4.25 4.33 (m, 3H, H3', H5'), $5.13 \sim 5.18\left(\mathrm{~m}, 1 \mathrm{H}, \mathrm{H} 4^{\prime}\right), 6.16$ (t, $J=6.8 \mathrm{~Hz}, 1 \mathrm{H}, \mathrm{H1}$ ), 6.59 (d, $J=14.4 \mathrm{~Hz}, 1 \mathrm{H}$, vinyl-H1), 6.86 (d, $J=13.6 \mathrm{~Hz}, 1 \mathrm{H}$, vinyl-H2), 7.70 (s, $1 \mathrm{H}, \mathrm{H} 6) ;{ }^{13} \mathrm{C}$ NMR $\left(100 \mathrm{MHz}, \mathrm{CDCl}_{3}\right)$ $\delta: 13.3,20.8,20.9,38.9,63.5,73.9,83.1,87.2,108.7$, 114.0, 128.1, 135.4, 152.6, 170.3, 170.4, 176.4. HRMS calcd for $\mathrm{C}_{16} \mathrm{H}_{19} \mathrm{BrN}_{2} \mathrm{O}_{6} \mathrm{SNa}[\mathrm{M}+\mathrm{Na}]^{+}: 469.0045$, found 469.0028 .

3.2.5 (E)-5-(2-溴乙烯基)-2'-脱氧胞嘧啶核苷(7)的合 成

将 $6(0.2 \mathrm{mmol})$ 、异丙醇 $(2 \mathrm{~mL})$ 和氨水 $(26 \%, 4 \mathrm{~mL})$ 依次加入到反应管中, 用旋塞密封后置入 $90{ }^{\circ} \mathrm{C}$ 油浴中 搅拌反应. 待 TLC 检测反应完全后, 减压浓缩, 残留物 用二氯甲烷/甲醇 $(V: V=10: 1)$ 作洗脱剂进行柱层析分 离得产物 7, 收率 74\%. ${ }^{1} \mathrm{H}$ NMR $\left(400 \mathrm{MHz}, \mathrm{DMSO}-d_{6}\right) \delta$ : $2.03 \sim 2.14\left(\mathrm{~m}, 2 \mathrm{H}, \mathrm{H} 2^{\prime}\right), 3.53 \sim 3.64\left(\mathrm{~m}, 2 \mathrm{H}, \mathrm{H} 5^{\prime}\right), 3.76$ (d, $\left.J=2.8 \mathrm{~Hz}, 1 \mathrm{H}, \mathrm{H} 3^{\prime}\right), 4.20$ (s, 1H , H3'-OH), 5.12 (s, 1H, H5'-OH), 5.20 (d, $\left.J=3.6 \mathrm{~Hz}, 1 \mathrm{H}, \mathrm{H} 4^{\prime}\right), 6.10$ (t, $J=6.8 \mathrm{~Hz}$, 1H, H1'), 6.71 (d, $J=13.6 \mathrm{~Hz}, 1 \mathrm{H}$, vinyl-H1), 7.03 (d, $J=$ $13.6 \mathrm{~Hz}, 1 \mathrm{H}$, vinyl-H2), 8.10 (s, $1 \mathrm{H}, \mathrm{H} 6) ;{ }^{13} \mathrm{C}$ NMR $(100$ MHz, DMSO- $\left.d_{6}\right) \delta: 41.1,61.2,70.2,85.7,87.7,100.9$, 106.1, 129.0, 138.9, 154.7, 163.0. HRMS calcd for $\mathrm{C}_{11} \mathrm{H}_{14} \mathrm{BrN}_{3} \mathrm{O}_{4} \mathrm{Na}[\mathrm{M}+\mathrm{Na}]^{+}:$354.0066, found 354.0060.
3.2.6 (E)-5-(2-溴乙烯基)-2'-脱氧- $N$-甲基胞嘧啶核苷 (8) 的合成

将 $6(0.2 \mathrm{mmol})$ 、异丙醇 $(2 \mathrm{~mL})$ 和甲氨水溶液 $(40 \%$, $4 \mathrm{~mL}$ ) 依次加入到反应管中, 用旋塞密封后置入 $90{ }^{\circ} \mathrm{C}$ 油浴中傥拌反应. 待 TLC 检测反应完全后, 减压浓缩, 残留物用二氯甲烷/甲醇 $(V: V=20: 1)$ 作洗脱剂进行柱 层析分离得产物 8, 收率 76\%. ${ }^{1} \mathrm{H}$ NMR $(400 \mathrm{MHz}$, DMSO- $\left.d_{6}\right) \delta: 2.04 \sim 2.13\left(\mathrm{~m}, 2 \mathrm{H}, \mathrm{H} 2^{\prime}\right), 2.77(\mathrm{~d}, J=4.0 \mathrm{~Hz}$, $\left.3 \mathrm{H}, \mathrm{CH}_{3}\right), 3.54 \sim 3.63\left(\mathrm{~m}, 2 \mathrm{H}, \mathrm{H} 5^{\prime}\right), 3.77(\mathrm{~d}, J=3.2 \mathrm{~Hz}$, 1H, H3'), 4.22 (t, $\left.J=4.0 \mathrm{~Hz}, 1 \mathrm{H}, \mathrm{H} 3^{\prime}-\mathrm{OH}\right), 5.12$ (t, $J=4.8$ $\left.\mathrm{Hz}, 1 \mathrm{H}, \mathrm{H} 4{ }^{\prime}\right), 6.13$ (t, $\left.J=6.4 \mathrm{~Hz}, 1 \mathrm{H}, \mathrm{H} 1^{\prime}\right), 6.74$ (d, $J=$ $13.2 \mathrm{~Hz}, 1 \mathrm{H}$, vinyl-H1), $7.00(\mathrm{~d}, J=13.2 \mathrm{~Hz}, 1 \mathrm{H}$, vinyl-H2), 7.54 (d, $J=4.0 \mathrm{~Hz}, 1 \mathrm{H}, \mathrm{NH}), 8.03(\mathrm{~s}, 1 \mathrm{H}, \mathrm{H6})$; ${ }^{13} \mathrm{C}$ NMR (100 MHz, DMSO- $\left.d_{6}\right) \delta: 28.1,41.0,61.3,70.2$, $85.5,87.7,104.1,106.8,128.7,137.7,154.6,160.7$. HRMS calcd for $\mathrm{C}_{12} \mathrm{H}_{16} \mathrm{BrN}_{3} \mathrm{O}_{4} \mathrm{Na}[\mathrm{M}+\mathrm{Na}]^{+}: 368.0222$, found 368.0216 .

辅助材料(Supporting Information) 化合物 $\mathbf{3 a} \sim 3 \mathbf{3}$, $\mathbf{4 a} \sim \mathbf{4 d}$ 和 $5 \sim 8$ 的 ${ }^{1} \mathrm{H} N M R 、{ }^{13} \mathrm{C}$ NMR 谱图. 这些材料 可以免费从本刊网站(http://sioc-journal.cn/)上下载.

\section{References}

[1] (a) Jordheim, L. P.; Durantel, D.; Zoulim, F.; Dumontet, C. Nat. Rev. Drug Discovery 2013, 12, 447.

(b) Wainberg, M. A. Antiviral Res. 2009, 81, 1.

(c) Jin, Y.-Y.; Xiao, Q.; Jv, Y. Chin. J. Org. Chem. 2009, 29, 44 (in Chinese).

(靳玄烨, 肖强, 巨勇, 有机化学, 2009, 29, 44.)

(d) Menéndez-Arias, L.; Álvarez, M.; Pacheco, B. Curr. Opin. Virol. 2014, 8,1 .

[2] (a) Xia, Y.; Liu, Y.; Rocchi, P.; Wang, M.; Fan, Y.; Qu, F.; Iovanna, J. L.; Peng, L. Cancer Lett. 2012, 318, 145.

(b) Wu, J.; Yu, W.; Fu, L.; He, W.; Wang, Y.; Chai, B.; Song, C.; Chang, J. Eur. J. Med. Chem. 2013, 63, 739.

(c) Shirouzu, H.; Morita, H.; Tsukamoto, M. Tetrahedron 2014, 70, 3635 .

[3] (a) Wainberg, M. A. Antiviral Res. 2009, 81, 1.

(b) De Clercq, E. Rev. Med. Virol. 2009, 19, 287.

(c) De Clercq, E. J. Med. Chem. 2010, 53, 1438.

(d) De Clercq, E. Antiviral Res. 2010, 85, 19.

[4] Bleackley, R. C.; Jones, A. S.; Walker, R. T. Tetrahedron 1976, 32 , 2795.

[5] Ashwell, M.; Jones, A. S.; Kumar, A.; Sayers, J. R.; Walker, R. T.; Sakuma, T.; de Clercq, E. Tetrahedron 1987, 43, 4601.

[6] Wang, C. J.; Xie, F.; Zhang, W. B. Chin. J. Org. Chem. 2008, 28, 503 (in Chinese).

(王春娟，谢芳，张万斌，有机化学, 2008, 28, 503.)

[7] Chelucci, G. Chem. Rev. 2012, 112, 1344 and references cited therein.

[8] (a) Hirao, T.; Masunaga, T.; Ohshiro, Y.; Agawa, T. J. Org. Chem. 1981, 46, 3745.

(b) Abbas, S.; Hayes, C. J.; Worden, S. Tetrahedron Lett. 2000, 41, 3215 . 
[9] Fan, X. S.; Li, P. Y.; Li, K.; Zhang, X. Y.; Zhao, W. Chin. J. Org. Chem. 2014, 34, 999 (in Chinese).

(范学森, 李培源, 李坤, 张新迎, 赵婉, 有机化学, 2014, 34, 999.)

[10] Corey, E. J.; Fuchs, P. L. Tetrahedron Lett. 1972, 13, 3769.

[11] (a) Wang, S. M.; Li, J.; Li, H. Y.; Liu, H. M.; Li, W. Chin. J. Org.
Chem. 2008, 28, 120 (in Chinese).

(王少敏，李娟，李华雨，刘宏民，李雯，有机化学， 2008，28, 120.)

(b) Li, W.; Liu, H.-M.; You, Q.-D. Acta Chim. Sinica 2003, 61, 1516 (in Chinese).

(李雯, 刘宏民, 尤启东, 化学学报, 2003, 61, 1516.)

(Qin, X.) 